\title{
Traduire
}

Revue française de la traduction

$239 \mid 2018$

Traduire l'art

\section{Dans les coulisses des musées et galeries d'art}

\section{Eleanor Madelaine}

\section{(2) OpenEdition \\ Journals}

Édition électronique

URL : http://journals.openedition.org/traduire/1522

DOI : 10.4000/traduire.1522

ISSN : 2272-9992

Éditeur

Société française des traducteurs

Édition imprimée

Date de publication : 1 décembre 2018

Pagination : 44-46

ISSN : 0395-773X

\section{Référence électronique}

Eleanor Madelaine, «Dans les coulisses des musées et galeries d'art », Traduire [En ligne], 239 | 2018, mis en ligne le 01 décembre 2018, consulté le 29 juin 2019. URL : http://journals.openedition.org/ traduire/1522 ; DOI : 10.4000/traduire.1522 


\section{Dans les coulisses des musées et galeries d'art}

\section{Eleanor Madelaine}

Pour les visiteurs des galeries d'art et des musées, les textes des cartels, des panneaux explicatifs ou des catalogues d'exposition sont avant tout une source d'information, un éclairage sur la vision de tel ou tel artiste, une mise en contexte des œuvres... Pour le traducteur, le style littéraire, voire poétique, de ce type de textes requiert une solide connaissance des techniques artistiques et de l'histoire de l'art tout autant qu'une jolie plume. Le public est loin de percevoir tout ce que cachent l'industrie de l'art et la traduction en art. Lorsque vous lisez l'expression "Prêt de ... " sur le cartel d'une œuvre d'art, vous êtes-vous déjà demandé comment cet objet est arrivé là et d'où il vient ? Laissez-vous guider dans les coulisses des musées et galeries d'art pour partager mon expérience de traductrice dans cet univers.

Pour moi, tout a commencé avec l'affaire familiale, une entreprise assurant le transport d'œurres d'art et fournissant divers services techniques aux musées et aux galeries d'art. J'ai grandi en entendant parler de prêts de musées, de caisses d'emballage et de licences d'exportation, sujets de conversation favoris à table ! Si j'ai suivi ma propre voie dans l'étude des langues étrangères et la traduction, je me suis naturellement spécialisée dans ce secteur lorsque je me suis installée à mon compte. C'est à l'occasion de mes tout premiers travaux dans ce domaine que j'ai pris conscience du fait que non seulement il est important de disposer de connaissances spécialisées, mais que ces traductions s'apparentent à bien des égards à la traduction technique ou juridique.

Examinons de plus près les différents types de textes à traduire dans ce domaine et, ce faisant, les défis que le traducteur doit relever.

Pour leurs expositions, les musées et les galeries empruntent des œuvres d'art à d'autres institutions ou à des collections privées. Ce processus de prêts et d'emprunts implique une multitude de documents : demandes de prêts, rapports sur les conditions d'exposition (prouvant que l'institution emprunteuse dispose des installations adéquates pour recevoir l'objet d'art), constat d'état (qui détermine l'état de l'œuvre avant son transfert), accord de prêt et conditions de prêt (qui précisent les dispositions prises inhérentes au prêt et le contrat passé 
entre les deux parties) et documents de transport (pertinents pour l'importation et l'exportation desdites œuvres d'art). Dans le cas de prêts internationaux, l'intervention du traducteur est indispensable pour favoriser une communication parfaite entre les parties.

Alors que la traduction des catalogues d'exposition et autres dépliants du même ordre exige une expérience en art et des connaissances en histoire de l'art, le traducteur intéressé par le travail dans les coulisses des musées et des galeries d'art ne doit pas nécessairement être un spécialiste de l'art ; pour autant, il doit maîtriser suffisamment le vocabulaire technique et la terminologie spécifique afin de rendre un travail de qualité. Le rapport sur les conditions d'exposition, par exemple, est un document rédigé par l'emprunteur et soumis au prêteur préalablement à la signature du prêt. II précise les conditions dans lesquelles l'œuvre d'art sera exposée et les mesures à prendre pour assurer sa protection et va jusqu'à donner des informations sur la structure du bâtiment, le système de sécurité, le système anti-incendie et la régulation du climat (température, hygrométrie...) dans les salles d'exposition. Lors de mon premier travail sur le sujet, j'ai ainsi dû chercher le nom des différents systèmes d'alarme tout comme les termes techniques liés à la régulation climatique des espaces d'exposition.

Une fois le prêt confirmé, le prêteur et l'emprunteur rédigent l'accord et les conditions du prêt. L'accord indique l'objet à prêter, les conditions dans lesquelles se fera le prêt et les responsabilités respectives des acteurs. Les conditions de prêt sont établies par l'institution prêteuse ; ce document juridique est rédigé dans un langage spécifique et dans un style très contraint qui implique de disposer de compétences en traduction juridique, mais aussi de s'assurer qu'on utilise la terminologie usuelle dans le milieu des musées et galeries d'art, attendue par les professionnels du secteur.

Avant qu'une œuvre quitte les locaux du prêteur, l'institution prêteuse établit un constat dit d'état qui en décrit l'état physique avant transfert : le conservateur, le plus souvent, recense alors les moindres traces d'usure et de dégradation. Ce document contient de nombreux termes techniques ; la tâche du traducteur est alors d'autant plus aisée qu'il est familier du domaine. À l'occasion d'une traduction d'un constat d'état pour une tapisserie du xive siècle, j'ai été confrontée à la terminologie complexe utilisée dans le domaine de la conservation des textiles, qui m'était étrangère. II m'a donc fallu procéder à des recherches minutieuses pour chacun des termes. Ce projet, chronophage mais intéressant, m'aura tout de même permis d'acquérir un grand nombre de termes spécifiques qui m'ont été très utiles par la suite.

Si la procédure du prêt d'œuvre est la même pour l'ensemble du secteur des musées et galeries d'art, chaque établissement est unique, ce qui rend le travail du traducteur varié et stimulant.

Comme pour tout domaine de spécialité, il est indispensable de se tenir informé des évolutions. Heureusement, de nombreux magazines ou revues, tel le Museums Journal publié par l'Association des musées du Royaume-Uni (Museums Association), constituent de précieuses ressources; on y trouve du vocabulaire technique et des explications sur le mode de 
fonctionnement des musées et galeries. L'idéal reste toutefois d'acquérir de l'expérience en travaillant sur le terrain afin d'appréhender ce fonctionnement de l'intérieur.

Une chose est sûre : si vous décidez de passer dans les coulisses des musées et galeries d'art, plus jamais vous ne visiterez une exposition ni ne contemplerez une œuvre d'art de la même manière!

eleanor@madelainetraduction.com

Originaire du nord de l'Angleterre, Eleanor Madelaine est diplômée en langue française et en traduction. Voilà dix ans qu'elle a emménagé en France dans le cadre de ses études, à l'issue desquelles elle a enseigné l'anglais pendant sept ans. Elle s'est installée il y a trois ans comme traductrice indépendante, profession qu'elle exerce désormais à plein temps. S'inspirant de ses expériences et de sa passion pour l'art et la culture, elle s'est spécialisée dans les domaines de la culture, du patrimoine, du tourisme et de la gastronomie.

\section{Webographie}

Tate Gallery, Loan agreement guidelines, https://www.tate.org.uk/about-us/projects/mattersmedia-art/lending-time-based-media-2005/loan-agreement-guidelines, consulté le 6 octobre 2018.

\section{Autres sites internet utiles}

Glossaire de I'Institut canadien de conservation (ICC) pour la rédaction de constats d'état : https://www.canada.ca/fr/institut-conservation/services/publications-conservation-preservation/ notes-institut-canadien-conservation/constat-etat-tableaux-glossaire.html

Museums Association (Association des musées du Royaume-Uni) :

https://www.museumsassociation.org 